\title{
ICSI significantly improved the pregnancy rate of patients with a high sperm DNA fragmentation index
}

\author{
Hee-Jun Chi', Seok-Gi Kim 1,2, Youn-Young Kim', Ji-Young Park', Chang-Seok Yoo', II-Hae Park', Hong-Gil Sun', Jae-Won Kim', \\ Kyeong-Ho Lee', Hum-Dai Park ${ }^{2}$
}

${ }^{1}$ IVF Center, Mamapapa and Baby Clinic, Ulsan; ${ }^{2}$ Department of Biotechnology, Daegu University, Daegu, Korea

Objective: Correlations between semen parameters and sperm DNA fragmentation index (DFI) were investigated to identify characteristics of sperm without DNA damage that could be used in selecting sperm for intracytoplasmic sperm injection (ICSI). Pregnancy outcomes were compared to determine whether in vitro fertilization (IVF) or ICSI is a better choice for patients who have sperm with a high-DFI.

Methods: Semen analysis was carried out in 388 patients who visited our IVF center for the first time to investigate correlations between sperm DFI and semen parameters. In addition, 1,102 IVF cycles in 867 patients were carried out in the present study; 921 cycles in the low-DFI group (DFI $<30 \%$ ) and 181 cycles in the high-DFI group (DFI $\geq 30 \%$ ). Both the low- and high-DFI groups were subdivided into IVF and ICSI cycle groups. Results: Sperm DFI showed significant inverse correlations with sperm motility $(r=-0.435, p<0.001)$ and morphology $(r=-0.153, p<0.05)$. Sperm DFI also showed significant correlations with rapid motility $(r=-0.436, p<0.001)$, and the kinetic parameters of average-path velocity $(r=-0.403)$ and linearity $(r=-0.412)$. Although there was no significant difference in the pregnancy rates between IVF $(48.6 \%)$ and ICSI $(44.8 \%)$ in the low-DFI group, the pregnancy rate of ICSI cycles $(44.8 \%, p<0.05)$ was significantly higher than IVF cycles $(25.0 \%)$ in the high-DFI group. No significant difference was observed in the abortion rates between the low-DFI (52 of 921,5.6\%) and high-DFI groups (7 of 181, 3.8\%). Conclusion: ICSI is a better choice than IVF for improving the pregnancy outcomes of patients who have sperm with a high DFI.

Keywords: Sperm DNA fragmentation index; Sperm injections, intracytoplasmic; Sperm morphology; Sperm motility

\section{Introduction}

Sperm DNA fragmentation may occur through one of the following potential causes: (1) defective chromatin condensation may occur during spermiogenesis [1]; (2) apoptosis may occur during spermatogenesis; and (3) oxidative stress may occur during the transit period through the male genital tract [2]. Although spermatozoa have no ability to repair damaged DNA, oocytes and embryos have a DNA repair system. Nevertheless, if the damage is severe, to the point that the repair system cannot fix it, embryonic development

Received: Dec 24, 2016 · Revised: Jun 16, 2017· Accepted: Jul 5, 2017

Corresponding author: Hee-Jun Chi

IVF Center, Mamapapa and Baby Clinic, 247 Samsan-ro, Nam-gu, Ulsan 44707, Korea

Tel:+81-52-258-6006 Fax:+81-52-258-6026 E-mail: hanna129@hanmail.net

This is an Open Access article distributed under the terms of the Creative Commons Attribution Non-Commercial License (http://creativecommons.org/licenses/by-nc/4.0/) which permits unrestricted non-commercial use, distribution, and reproduction in any medium, provided the original work is properly cited. can be arrested [3].

Various methods are available to assess sperm DNA integrity, including the single cell gel electrophoresis assay [4], terminal deoxynucleotidyl transferase-mediated nick end labelling [5], the sperm chromatin structure assay [6], and the sperm chromatin dispersion test [7]. The sperm chromatin dispersion test has been recognized as a much simpler and economical test than other tests, because it does not require expensive equipment such as flow cytometry or fluorescent microscopy.

To minimize sperm DNA fragmentation and/or to select sperm without DNA fragmentation for assisted reproductive technology (ART), several strategies have been proposed: the intake of oral antioxidants [8], varicocele repair [9], intracytoplasmic morphologically selected sperm injection [10], hyaluronic acid-bound sperm selection [11,12], the electrophoretic separation technique [13], density gradient centrifugation (DGC) [14-16], magnetic-activated cell sorting [17], and the combination of DGC and magnetic activated cell sorting [18-20]. 
Various thresholds of the DNA fragmentation index (DFI) have been used in previous studies: 15\% [21], 19\% [22], 27\% [23,24], 30\% [2528], 35\% [29], and 44\% [30]. The different threshold values among the studies may have resulted from the use of different DFI detection methods, patient populations, type of fertilization (in vitro fertilization [IVF] or intracytoplasmic sperm injection [ICSI]), or the sperm preparation method (swim-up or DGC) for the ART procedures [31]. Recently, it has become generally accepted that a meaningful cutoff value of the sperm DFI is $30 \%$ because DFI values lower than $30 \%$ have been found to be strongly associated with natural conception and success in intrauterine insemination [32], and values above this cutoff have been reported to be associated with lower rates of pregnancy and delivery $[25,27,28]$.

Several studies have demonstrated that sperm DFI has significant correlations with the sperm parameters of concentration [25,33], morphology [4,20,33-35], and motility [4,20,35,36]. However, some authors did not observe significant correlations between sperm DFI and morphology [37,38], concentration [20,34], and motility [34]. Moreover, the effect of a high DFI upon the clinical outcomes of IVF programs is still controversial. Many authors have observed correlations between the DFI and clinical outcomes, including low fertilization rates [39,40], embryo quality and development [26,41-44], implantation [22], pregnancy rates $[30,45]$ and increased pregnancy loss [46-48]. In contrast, other authors have found no significant relationship between the DFI and pregnancy outcomes from IVF or ICSI [23,27,49-51]. In addition, although Collins et al. [52] and the Practice Committee of the American Society for Reproductive Medicine [53] reported that DNA damage analysis was insufficiently predictive of pregnancy outcomes to justify its routine use in the evaluation of infertility, many clinicians and embryologists still believe that evaluating sperm DNA integrity makes a meaningful contribution to the semen analysis profile used for both diagnostic and prognostic purposes [54].

Debate also exists regarding whether ICSI or conventional IVF is preferable for cases of sperm with a high DFI. The widespread use of ICSI in treating couples with severe male factor infertility may result in DNA-damaged sperm mistakenly being injected into the oocyte, with potentially hazardous consequences [55]. This can occur because spermatozoa with apparently normal motility and morphology may carry fragmented DNA in their nucleus [56] and can fertilize oocytes [57]. In addition, sperm with the potential to penetrate the cumulus oophorus layer and to fertilize the oocyte were found to have greater DNA integrity than those that could not when IVF was performed [23]. On the contrary, when performing ICSI, embryologists attempt to select spermatozoa with normal morphology and rapid motility, and it has been shown that preselected sperm tend to have less DNA damage $[10,58]$. Therefore, ICSI is regarded as a pro- cess that decreases the likelihood of DNA fragmentation in spermatozoa [59-61].

Many authors have conducted studies to assess possible associations between sperm DFI and clinical outcomes in ICSI cycles. Some scientists have reported that sperm DFI in ICSI cycles was not correlated with clinical outcomes, including fertilization rate [22,29,51,62], embryo quality and development [22,29], the pregnancy rate [22,51], the live birth rate [61], and pregnancy loss [22]. On the contrary, other scientists have reported that high DFI significantly decreased the fertilization rate [21,63], embryo quality and development [62-67], the pregnancy rate $[62,64,67]$, and ongoing pregnancy [68-70], as well as increasing the pregnancy loss rate $[21,27,28,41,47,62]$. In addition, although some authors have reported that there was no difference in the pregnancy outcomes of the high-DFI group between IVF and ICSI cycles [29,42], other authors have observed beneficial effects of ICSI on clinical outcomes, including the fertilization rate $[21,71]$, pregnancy rate $[23,27,28,51,71]$, and delivery rate $[23]$, compared to IVF. Thus, these results suggest that ICSI may be preferable in cases with a high DFI $[23,28,51,61,71,72]$. However, the selection of IVF or ICSI for sperm with a high DFI remains an unclear and difficult problem awaiting resolution.

This study was conducted to investigate whether IVF or ICSI is a better choice for patients who have sperm with a high DFI. In this study, correlations between semen parameters and sperm DFI were investigated to identify the characteristics of sperm with high DNA integrity for selecting sperm in ICSI cycles, and pregnancy outcomes were compared between IVF and ICSI cycles in both low- and highDFI groups.

\section{Methods}

This was a retrospective study approved by the Institutional Review Board of Mamapapa and Baby Clinic (IRB No. 2015-02). This study was carried out from April 2015 to March 2017.

\section{Semen analysis and sperm chromatin dispersion test}

Semen analysis and the Halosperm test were carried out in 388 patients who visited IVF center of Mamapapa and Baby Clinic for the first time. Their semen analysis data and sperm DFI values were used to analyze correlations between sperm DFI and semen parameters. For semen analysis, semen samples were collected by masturbation after 3 to 7 days of sexual abstinence. After the liquefaction of semen at room temperature, semen analysis was performed according to World Health Organization (WHO) guidelines (2010) with computerassisted semen analysis (CASA). The sperm chromatin dispersion test using the Halosperm kit (Halotech DNA, Madrid, Spain) was carried out according to the process described by Chi et al. [20]. In addition to 
the 388 new patients, the Halosperm test was also carried out in the semen samples of 867 IVF patients on ovum pick-up day to determine whether IVF or ICSI would be used as the fertilization method.

\section{ART program}

A total of 1,102 cycles from 867 IVF patients were included in the present study, of which 921 cycles belonged to the low-DFl group (DFI <30\%) and 181 cycles belonged to the high-DFI group (DFI $\geq 30 \%$ ). Both the low- and high-DFI groups were subdivided into IVF and ICSI cycle groups. In the low-DFI group, 343 IVF and 578 ICSI cycles were conducted, while 36 IVF and 145 ICSI cycles were conducted in the high-DFI group. ICSI was used in patients who showed various manifestations of male factor infertility, including high sperm DNA damage; a low number of oocytes aspirated; and/or a low fertilization rate in previous IVF cycles. The clinical outcomes of the groups were compared. Clinical pregnancy was confirmed by ultrasound confirmation of a gestational sac or heartbeat.

\section{Sperm preparation}

Semen samples of the patients were treated with DGC using SpermGrad (Vitrolife, Gothenburg, Sweden) after liquefaction. This discontinuous density gradient consisted of two (90\% and $45 \%$ ) $1-\mathrm{mL}$ layers of SpermGrad, and 1 to $3 \mathrm{~mL}$ of semen was deposited on the $45 \%$ layer. The gradient was then centrifuged at $400 \mathrm{~g}$ for 15 minutes. After centrifugation, the seminal plasma supernatant was discarded, and the sperm pellet was washed twice with $10 \mathrm{~mL}$ of Ham's F-10 medium (Gibco; Life Technologies, New York, NY, USA) supplemented with $0.5 \mathrm{~mL}$ of synthetic serum substitute (Irvine Scientific, Santa Ana, CA, USA) by centrifugation. The sperm pellet was washed with 1 $\mathrm{mL}$ of embryo culture medium (SAGE 1-Step; Origio, Malov, Denmark), and then the pellet was resuspended with the culture medium. Based on our previous results, ICSI was performed for sperm showing a high movement velocity and normal morphology. However, motility was the first consideration and morphology was the second consideration for the selection of sperm for ICSI, because the DFI showed a significantly higher correlation with motility than with morphology in our previous study.

\section{Embryo quality scoring}

Both conventional IVF and ICSI embryos were scored tat the following three time points after fertilization: 2-cell cleavage (26 hours), day 2 (40-41 hours), and day 3 (64-65 hours). The total summed score of the highest-quality embryo was 12 points, if the embryo showed 2-cell cleavage ( +2 points), morphological grade 1 on day $2(+5$ points), and morphological grade 1 on day 3 ( +5 points). The score was dependent on the morphological grade and cleavage speed of embryos. The morphological grades of embryos were used to score them from grade 1 to 5 according to the number and shape of blastomeres and degree of fragmentation. The characteristics of morphological grades and scores were as follows: grade 1 (5 points), no fragmentation with equal-sized blastomeres; grade 2 (4 points), $<10 \%$ fragmentation with equal-sized blastomeres or no fragmentation with unequal-sized blastomeres; grade 3 (3 points), 10\% $\leq$ fragmentation <25\%; grade 4 ( 2 points), $25 \% \leq$ fragmentation $<50 \%$; and grade 5 (1 point), $\geq 50 \%$ fragmentation. In addition, when the embryos showed a slow developmental speed, a point was subtracted from the grade $(-1)$. For example, the score of a 2 -cell stage (grade 1 ) embryo on day 2 would be 4 points ( $5-1=4$ points). The mean embryo quality score was calculated by dividing the $\mathrm{cu}$ mulative embryo scores of transferred embryos by the number of transferred embryos.

\section{Statistical analysis}

Statistical analysis was performed with SPSS ver. 11.0 for Windows (SPSS Inc., Chicago, IL, USA). Means and standard deviations were calculated for all variables. To assess the relationships of sperm DFI with semen parameters, motility velocity, and kinetic parameters of motility, the Pearson correlation coefficient was used. One-way analysis of variance was used to compare the characteristics of patients and clinical outcomes between the subgroups (IVF and ICSI cycles) of the low- and high-DFI groups. The rates of pregnancy and abortion in the sub-groups were compared using the chi-square test. The $p$ values $<0.05$ were considered to indicate statistical significance.

\section{Results}

We conducted semen analysis and the Halosperm assay in the semen samples of 388 patients who visited our IVF center for the first time to investigate correlations between sperm DFI and semen parameters (Table 1). Although sperm DFI showed significant inverse correlations with sperm motility $(r=-0.435, p<0.001)$ and morphology $(r=-0.153, p<0.05)$, the DFI did not show a meaningful correlation with the patients' age $(r=0.098)$, semen volume $(r=0.045)$, or

Table 1. Correlations between the sperm DFI and semen parameters in 388 semen samples

\begin{tabular}{lccc}
\hline Semen parameter & Mean \pm SD & $\begin{array}{c}\text { Correlation } \\
\text { coefficient with } \\
\text { sperm DFI }(r)\end{array}$ & $p$-value \\
\hline Male patients' age $(\mathrm{yr})$ & $38.0 \pm 4.4$ & 0.098 & $\mathrm{NS}$ \\
Volume $(\mathrm{mL})$ & $3.1 \pm 1.5$ & 0.045 & $\mathrm{NS}$ \\
Concentration $\left(10^{6} / \mathrm{mL}\right)$ & $100.3 \pm 97.5$ & 0.091 & $\mathrm{NS}$ \\
Motility $(\%)$ & $49.9 \pm 19.8$ & -0.435 & $<0.001$ \\
Morphology (\%) & $6.2 \pm 3.6$ & -0.153 & $<0.05$ \\
\hline
\end{tabular}

DFI, DNA fragmentation index; SD, standard deviation; NS, not significant. 
sperm concentration $(r=0.091)$. Therefore, sperm DFI showed the highest correlation with motility, in contrast to other parameters.

We investigated the correlation between sperm DFI and velocity of motile sperm (Table 2). Motile spermatozoa were classified into three groups (rapid, medium, and slow velocity) by the CASA system. Sperm DFI was significantly more strongly associated with rapid motility $(r=-0.436, p<0.001)$ than medium $(r=-0.122, p<0.05)$ or slow motility ( $r=-0.013$, not significant). These results indicated that sperm showing rapid velocity had greater DNA integrity than sperm with a medium or slow velocity.

We investigated the correlation between sperm DFI and the kinetic parameters of motility measured by CASA (Table 3). Sperm DFI showed a significant inverse correlation with all kinetic parameters. In particular, average-path velocity $(r=-0.403)$ and linearity $(r=-0.412)$ showed stronger correlations with the DFI than other kinetic parameters.

Table 4 summarizes the characteristics of 867 patients and the clinical outcomes of IVF and ICSI cycles (1,102 cycles) in the low- and high-DFI groups. The 1,102 IVF cycles were divided into low-DFI (921 cycles) and high-DFI (181 cycles) groups, and then both the low-and high-DFI groups were subdivided into IVF and ICSI cycles. In the lowDFI group, there were no significant differences in the patients' age,

Table 2. Correlations between the sperm DFI and the velocity of motile sperm

\begin{tabular}{lccc}
\hline Motility velocity & Mean \pm SD & $\begin{array}{c}\text { Correlation } \\
\text { coefficient with } \\
\text { sperm DFI }(r)\end{array}$ & $p$-value \\
\hline Progressive motility (\%) & $49.1 \pm 19.8$ & -0.436 & $<0.001$ \\
Rapid motility (\%) & $42.2 \pm 18.9$ & -0.436 & $<0.001$ \\
Medium motility (\%) & $6.9 \pm 3.2$ & -0.122 & $<0.050$ \\
Slow motility (\%) & $1.1 \pm 3.7$ & -0.013 & NS \\
\hline
\end{tabular}

DFI, DNA fragmentation index; SD, standard deviation; NS, not significant. endometrial thickness, number of oocytes aspirated, or quality and number of embryos transferred between IVF and ICSI cycles. Although the fertilization rate in the ICSI cycles $(87.0 \%, p<0.05)$ was significantly higher than that of the IVF cycles (78.4\%), the pregnancy rate in the ICSI cycles (44.8\%) did not significantly differ from that observed in the IVF cycles (48.6\%). Moreover, in the high-DFI group, there were no significant differences in the characteristics of patients between the IVF and ICSI patients. However, the DFI of the ICSI patients $(43.5 \pm 12.1, p<0.05)$ was significantly higher than that of the IVF patients (35.6 \pm 7.5$)$; nevertheless, the pregnancy rate in the ICSI cycles $(44.8 \%, p<0.05)$ was significantly higher than that in the IVF cycles (25.0\%). There was no significant difference in the abortion rate between the low- and high-DFI groups.

\section{Discussion}

In the present study, sperm DFI showed a significant inverse correlation with sperm motility $(r=-0.435, p<0.001)$ which is consistent with the results of many previous studies $[4,20,25,33,35,36,38]$. Only Brahem et al. [34] did not observe a significant correlation between

Table 3. Correlations between sperm DFI and kinetic parameters of motility

\begin{tabular}{lccc}
\hline Kinetic parameter & Mean \pm SD & $\begin{array}{c}\text { Correlation coefficient } \\
\text { with sperm DFI }(r)\end{array}$ & $p$-value \\
\hline VCL & $23.6 \pm 12.2$ & -0.393 & $<0.001$ \\
VSL & $10.6 \pm 5.5$ & -0.394 & $<0.001$ \\
VAP & $15.0 \pm 7.6$ & -0.403 & $<0.001$ \\
LIN=VSL/NCL & $23.5 \pm 9.8$ & -0.412 & $<0.001$ \\
STR=VSL/NAP & $70.7 \pm 8.5$ & -0.131 & $<0.050$ \\
ALH & $0.9 \pm 0.5$ & -0.382 & $<0.001$ \\
\hline
\end{tabular}

DFI, DNA fragmentation index; SD, standard deviation; $\mathrm{VCL}$, curvilinear velocity; VSL, straight-line velocity; VAP, average-path velocity; LIN, linearity; STR, straightness; ALH, amplitude of lateral head displacement.

Table 4. Characteristics of 867 patients and clinical outcomes of 1,102 IVF and ICSI cycles classified with sperm DFI

\begin{tabular}{|c|c|c|c|c|}
\hline \multirow{2}{*}{ Variable } & \multicolumn{2}{|c|}{ Low-DFI group (DFI <30\%) } & \multicolumn{2}{|c|}{ High-DFI group (DFI $\geq 30 \%$ ) } \\
\hline & IVF cycle $(n=343)$ & ICSI cycle $(n=578)$ & IVF cycle $(n=36)$ & ICSI cycle $(n=145)$ \\
\hline DFI (\%) & $14.1 \pm 6.2^{\mathrm{a}}$ & $14.9 \pm 6.7^{\mathrm{a}}$ & $35.6 \pm 7.5^{\mathrm{b}}$ & $43.5 \pm 12.1^{c}$ \\
\hline Female age (yr) & $36.5 \pm 3.9^{\mathrm{a}}$ & $36.9 \pm 4.2^{\mathrm{a}}$ & $39.2 \pm 4.1^{\mathrm{b}}$ & $37.8 \pm 4.1^{\mathrm{b}}$ \\
\hline Endometrium (mm) & $9.9 \pm 2.3$ & $9.8 \pm 2.2$ & $9.6 \pm 2.6$ & $10.1 \pm 2.3$ \\
\hline No. of eggs aspirated & $8.9 \pm 4.8$ & $8.2 \pm 4.9$ & $6.9 \pm 4.4$ & $8.1 \pm 4.2$ \\
\hline Fertilization rate (\%) & $78.4^{\mathrm{a}}$ & $87.0^{\mathrm{b}}$ & $86.9^{\mathrm{b}}$ & $85.5^{\mathrm{b}}$ \\
\hline No. of ET & $1.9 \pm 0.3$ & $1.8 \pm 0.3$ & $1.9 \pm 0.3$ & $1.8 \pm 0.4$ \\
\hline Embryo quality score & $8.2 \pm 3.2$ & $8.3 \pm 3.2$ & $8.5 \pm 3.1$ & $8.2 \pm 3.1$ \\
\hline Clinical pregnancy & $167(48.6)^{a}$ & $254(44.8)^{\mathrm{a}}$ & $9(25.0)^{b}$ & $65(44.8)^{a}$ \\
\hline Abortion & $29(8.4)^{\mathrm{a}}$ & $23(3.9)^{b}$ & $2(5.5)^{\mathrm{a}, \mathrm{b}}$ & $6(4.1)^{\mathrm{a}, \mathrm{b}}$ \\
\hline
\end{tabular}

Values are presented as mean \pm standard deviation or number (\%). Different superscript letters indicate significant differences $(p<0.05)$. IVF, in vitro fertilization; ICSI, intracytoplasmic sperm injection; DFI, DNA fragmentation index; ET, embryos transferred.

${ }^{\text {a) }}$ Fertilization rate $(\%)=$ (number of fertilized oocytes/number of oocytes inseminated or that underwent ICSI) $\times 100$. 
DFI and motility $(r=0.051, p=0.741)$. This may have resulted from excessive variance across samples even though the number of semen samples was small ( 40 semen samples). The semen samples in that study showed wide ranges of semen parameters, as exemplified by age ( $27-54$ years), motility ( $5 \%-48 \%)$, and DFI ( $18 \%-72 \%)$. However, they found a significant decrease of DFI and increase of motility following DGC compared with raw semen, which means that sperm DFI has an inverse relationship with sperm motility. Therefore, it is accepted without doubt that sperm motility has a significant correlation with the DFI.

In the present study, sperm DFI also showed a significant inverse correlation with sperm morphology $(r=-0.153, p<0.05)$, although the coefficient was lower than was observed for sperm motility $(r=-0.435)$. In contrast to our results, some authors did not observe an inverse correlation between sperm DFI and morphology [37,38]. This may have resulted from differences in the cutoff values used for sperm morphology. Our cutoff value for sperm morphology was $4 \%$ (WHO 2010 guidelines), but they used the cutoff value of 30\% (WHO 1999 guidelines). Moreover, unlike motility and concentration of sperm, sperm morphology is generally assessed by using a manual protocol rather than by using a CASA program, because the CASA program revises long time for assessment. Therefore, there is a possibility of discrepancies in the evaluation of the sperm morphology between the authors due to the subjective estimations of embryologists. Nevertheless, many authors reported a significant correlation between sperm DFI and morphology [4,20,33-35].

We observed that sperm DFI showed a higher correlation with sperm motility than with other semen parameters. To analyze this correlation in greater depth, we investigated the correlation between sperm DFI and the velocity of motile sperm (Table 2). As we expected, sperm DFI showed a significantly stronger negative correlation with rapid motility than with medium or slow motility. This result suggests that selecting sperm with rapid motility may be an approach for selecting sperm with high DNA integrity. To investigate this correlation even more closely, we investigated the correlation between sperm DFI and the kinetic parameters of motility measured by CASA (Table 3). Although sperm DFI showed a significant inverse correlation with all kinetic parameters, the strongest correlations were observed with average-path velocity $(r=-0.403)$ and linearity $(r=-0.412)$ which means that sperm moving a long distance within a given time had high DNA integrity. Based on these results, we endeavored to select sperm with rapid motility and good morphology for ICSI. However, we gave priority to motility over morphology as a decision-making criterion for the selection.

In the present study, the fertilization rates in the IVF (86.9\%) and ICSI cycles (85.5\%) in the high-DFI group were significantly higher than the rate in the IVF cycles $(78.4 \%, p<0.05)$ and were similar to the rate of ICSI cycles (87.0\%) in the low-DFI group. These results are consistent with previous reports that a significant correlation was not found between DFI and the fertilization rate $[22,29,42,51,62]$. In contrast to these results, Oleszczuk et al. [71] reported that the fertilization rate (38.1\%) of IVF cycles was significantly lower than that (61\%) of ICSI cycles in patients with a high DFI. However, this discrepancy may have been caused by the inclusion of immature oocytes in IVF and the exclusion of immature oocytes in ICSI cycles, rather than by high DFI. Benchaib et al. [21] likewise reported a significant negative relationship between the fertilization rate and DFI in ICSI cycles. However, they reported that the fertilization rate of ICSI cycles was $0 \%$ when the sperm DFI was over $25 \%$. This fertilization rate is markedly lower than other reports. Therefore, these results indicate that sperm DNA damage does not exert a significant influence on the fertilization ability of sperm.

Although there was no difference in the number and quality of the embryos transferred or endometrial thickness between the IVF cycles of the low- and high-DFI groups, the pregnancy rate $(25.0 \%)$ of the IVF cycles in the high-DFI group was significantly lower than that (48.6\%) of their counterparts in the low-DFI group. This result is consistent with reports of a significant negative correlation between sperm DFI and pregnancy rate in IVF cycles [45,73]. However, the female partner's age (39.2 \pm 4.1 years) and the fertilization rate $(86.9 \%)$ of the IVF cycles in high-DFI group were significantly different from the corresponding values $(36.5 \pm 3.9$ years and $78.4 \%$, respectively; $p<0.05)$ of the IVF cycles in low-DFI group. Therefore, these differences may be a potential partial cause of the difference in the pregnancy rates. However, no significant difference was observed in the pregnancy rates of ICSI cycles between the low-DFI (44.8\%) and high-DFI (44.8\%) groups, which suggests that high sperm DFI was not related to the pregnancy rate in ICSI cycles. This result is similar to those of previous studies $[28,42,51]$.

Negative opinions have been expressed regarding ICSI for sperm with a high DFI, because DNA-damaged sperm may have a negative impact on the outcome of ICSI cycles in which DNA-damaged sperm is directly injected into the oocyte $[26,41,65]$. Moreover, in IVF cycles, the fertilizing spermatozoon may be selected by the zona pellucida, which can reject aneuploid candidates [74], and the zona pellucida play have a role in preventing DNA-damaged sperm binding, especially Y-bearing sperm [75]. However, recently, these concerns about ICSI have come to be regarded as a form of over-anxiety because a beneficial effect of ICSI on the pregnancy rate of patients with high sperm DFI has been reported by many authors $[23,27,28,51,71]$. In the present study, we likewise observed that the pregnancy rate of ICSI cycles (44.8\%) was significantly higher than the rate of IVF cycles (25.0\%) in the high-DFI group. The beneficial effect of ICSI can be explained by previous reports that poor morphology of spermatozoa is 
strongly associated with increased DNA fragmentation [76], and that sperm DFI shows a significant inverse correlation with sperm motility and morphology $[4,20,35]$. When performing ICSI, most embryologists attempt to select spermatozoa showing normal morphology and rapid motility, which is a kind of preselection of sperm with less DNA damage [10,58].

In the previous studies, a positive association between the spontaneous abortion rate and high DFI was reported in ICSI cycles [21,62] and both IVF and ICSI cycles $[28,47,51,77]$. However, we did not observe a significant correlation between DFI and abortion rates regardless of DFI value and type of ART program.

In conclusion, sperm DFI showed a significant inverse correlation with the proportions of sperm with rapid progressive motility and normal morphology. On the basis of this result, we tried to select spermatozoa showing normal morphology and rapid motility. There were no significant differences in the characteristics of patients or the pregnancy rate between IVF and ICSI cycles in the low-DFI group. However, in the high-DFI group, the pregnancy rate in the IVF cycles was significantly lower than in the ICSI cycles, although statistically significant differences were not found in the characteristics of patients, number of eggs, fertilization rate, or embryo quality between the two groups. Moreover, the DFI observed in the ICSI cycles in the high-DFI group was significantly higher than that of the ICSI cycles in the low-DFI group. Nevertheless, there was no significant difference in the pregnancy rate between the two groups. Therefore, a decrease in the pregnancy rate due to sperm DNA fragmentation can be prevented by ICSI.

\section{Conflict of interest}

No potential conflict of interest relevant to this article was reported.

\section{References}

1. Bianchi PG, Manicardi GC, Urner F, Campana A, Sakkas D. Chromatin packaging and morphology in ejaculated human spermatozoa: evidence of hidden anomalies in normal spermatozoa. Mol Hum Reprod 1996;2:139-44.

2. Muratori M, Tamburrino L, Marchiani S, Cambi M, Olivito B, Azzari $C$, et al. Investigation on the origin of sperm DNA fragmentation: role of apoptosis, immaturity and oxidative stress. Mol Med 2015;21:109-22.

3. Gawecka JE, Marh J, Ortega M, Yamauchi Y, Ward MA, Ward WS. Mouse zygotes respond to severe sperm DNA damage by delaying paternal DNA replication and embryonic development. PLoS One 2013;8:e56385.

4. Chi HJ, Chung DY, Choi SY, Kim JH, Kim GY, Lee JS, et al. Integrity of human sperm DNA assessed by the neutral comet assay and its relationship to semen parameters and clinical outcomes for the IVF-ET program. Clin Exp Reprod Med 2011;38:10-7.

5. Sharma R, Ahmad G, Esteves SC, Agarwal A. Terminal deoxynucleotidyl transferase dUTP nick end labeling (TUNEL) assay using bench top flow cytometer for evaluation of sperm DNA fragmentation in fertility laboratories: protocol, reference values, and quality control. J Assist Reprod Genet 2016;33:291-300.

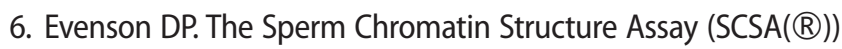
and other sperm DNA fragmentation tests for evaluation of sperm nuclear DNA integrity as related to fertility. Anim Reprod Sci 2016;169:56-75.

7. Fernandez JL, Cajigal D, Lopez-Fernandez C, Gosalvez J. Assessing sperm DNA fragmentation with the sperm chromatin dispersion test. Methods Mol Biol 2011;682:291-301.

8. Abad C, Amengual MJ, Gosalvez J, Coward K, Hannaoui N, Benet $J$, et al. Effects of oral antioxidant treatment upon the dynamics of human sperm DNA fragmentation and subpopulations of sperm with highly degraded DNA. Andrologia 2013;45:211-6.

9. Li F, Yamaguchi K, Okada K, Matsushita K, Ando M, Chiba K, et al. Significant improvement of sperm DNA quality after microsurgical repair of varicocele. Syst Biol Reprod Med 2012;58:274-7.

10. Berkovitz A, Eltes F, Yaari S, Katz N, Barr I, Fishman A, et al. The morphological normalcy of the sperm nucleus and pregnancy rate of intracytoplasmic injection with morphologically selected sperm. Hum Reprod 2005;20:185-90.

11. Parmegiani L, Cognigni GE, Bernardi S, Troilo E, Ciampaglia W, Filicori M. "Physiologic ICSI": hyaluronic acid (HA) favors selection of spermatozoa without DNA fragmentation and with normal nucleus, resulting in improvement of embryo quality. Fertil Steril 2010;93:598-604.

12. Yagci A, Murk W, Stronk J, Huszar G. Spermatozoa bound to solid state hyaluronic acid show chromatin structure with high DNA chain integrity: an acridine orange fluorescence study. J Androl 2010;31:566-72

13. Fleming SD, Ilad RS, Griffin AM, Wu Y, Ong KJ, Smith HC, et al. Prospective controlled trial of an electrophoretic method of sperm preparation for assisted reproduction: comparison with density gradient centrifugation. Hum Reprod 2008;23:2646-51.

14. Enciso M, Iglesias M, Galan I, Sarasa J, Gosalvez A, Gosalvez J. The ability of sperm selection techniques to remove single- or double-strand DNA damage. Asian J Androl 2011;13:764-8.

15. Rougier N, Uriondo H, Papier S, Checa MA, Sueldo C, Alvarez Sedo $C$. Changes in DNA fragmentation during sperm preparation for intracytoplasmic sperm injection over time. Fertil Steril 2013;100:69-74

16. Sa R, Cunha M, Rocha E, Barros A, Sousa M. Sperm DNA frag- 
mentation is related to sperm morphological staining patterns. Reprod Biomed Online 2015;31:506-15.

17. Said TM, Agarwal A, Grunewald S, Rasch M, Glander HJ, Paasch U. Evaluation of sperm recovery following annexin $\mathrm{V}$ magnetic-activated cell sorting separation. Reprod Biomed Online 2006;13: 336-9.

18. Lee TH, Liu CH, Shih YT, Tsao HM, Huang CC, Chen HH, et al. Magnetic-activated cell sorting for sperm preparation reduces spermatozoa with apoptotic markers and improves the acrosome reaction in couples with unexplained infertility. Hum Reprod 2010;25:839-46.

19. Tavalaee M, Deemeh MR, Arbabian M, Nasr-Esfahani MH. Density gradient centrifugation before or after magnetic-activated cell sorting: which technique is more useful for clinical sperm selection? J Assist Reprod Genet 2012;29:31-8.

20. Chi HJ, Kwak SJ, Kim SG, Kim YY, Park JY, Yoo CS, et al. Efficient isolation of sperm with high DNA integrity and stable chromatin packaging by a combination of density-gradient centrifugation and magnetic-activated cell sorting. Clin Exp Reprod Med 2016; 43:199-206.

21. Benchaib M, Lornage J, Mazoyer C, Lejeune H, Salle B, Francois Guerin J. Sperm deoxyribonucleic acid fragmentation as a prognostic indicator of assisted reproductive technology outcome. Fertil Steril 2007;87:93-100.

22. Speyer BE, Pizzey AR, Ranieri M, Joshi R, Delhanty JD, Serhal P. Fall in implantation rates following ICSI with sperm with high DNA fragmentation. Hum Reprod 2010;25:1609-18.

23. Bungum $M$, Humaidan $P$, Spano $M$, Jepson $K$, Bungum L, Giwercman $A$. The predictive value of sperm chromatin structure assay (SCSA) parameters for the outcome of intrauterine insemination, IVF and ICSI. Hum Reprod 2004;19:1401-8.

24. Boe-Hansen GB, Fedder J, Ersboll AK, Christensen P. The sperm chromatin structure assay as a diagnostic tool in the human fertility clinic. Hum Reprod 2006;21:1576-82.

25. Trisini AT, Singh NP, Duty SM, Hauser R. Relationship between human semen parameters and deoxyribonucleic acid damage assessed by the neutral comet assay. Fertil Steril 2004;82:1623-32.

26. Zini A, Meriano J, Kader K, Jarvi K, Laskin CA, Cadesky K. Potential adverse effect of sperm DNA damage on embryo quality after ICSI. Hum Reprod 2005;20:3476-80.

27. Bungum $M$, Humaidan $P$, Axmon $A$, Spano M, Bungum L, Erenpreiss J, et al. Sperm DNA integrity assessment in prediction of assisted reproduction technology outcome. Hum Reprod 2007; 22:174-9.

28. Zhao J, Zhang Q, Wang Y, Li Y. Whether sperm deoxyribonucleic acid fragmentation has an effect on pregnancy and miscarriage after in vitro fertilization/intracytoplasmic sperm injection: a sys- tematic review and meta-analysis. Fertil Steril 2014;102:9981005.e8.

29. Anifandis G, Bounartzi T, Messini Cl, Dafopoulos K, Markandona $R$, Sotiriou $S$, et al. Sperm DNA fragmentation measured by Halosperm does not impact on embryo quality and ongoing pregnancy rates in IVF/ICSI treatments. Andrologia 2015;47:295-302.

30. Simon L, Brunborg G, Stevenson M, Lutton D, McManus J, Lewis SE. Clinical significance of sperm DNA damage in assisted reproduction outcome. Hum Reprod 2010;25:1594-608.

31. Zhang Z, Zhu L, Jiang H, Chen H, Chen Y, Dai Y. Sperm DNA fragmentation index and pregnancy outcome after IVF or ICSI: a meta-analysis. J Assist Reprod Genet 2015;32:17-26.

32. Evenson DP, Wixon R. Data analysis of two in vivo fertility studies using sperm chromatin structure assay-derived DNA fragmentation index vs. pregnancy outcome. Fertil Steril 2008;90:1229-31.

33. Manochantr S, Chiamchanya C, Sobhon P. Relationship between chromatin condensation, DNA integrity and quality of ejaculated spermatozoa from infertile men. Andrologia 2012;44:187-99.

34. Brahem S, Mehdi M, Elghezal H, Saad A. Semen processing by density gradient centrifugation is useful in selecting sperm with higher double-strand DNA integrity. Andrologia 2011;43:196202.

35. Sivanarayana T, Krishna CR, Prakash GJ, Krishna KM, Madan K, Rani BS, et al. CASA derived human sperm abnormalities: correlation with chromatin packing and DNA fragmentation. J Assist Reprod Genet 2012;29:1327-34.

36. Varghese AC, Bragais FM, Mukhopadhyay D, Kundu S, Pal M, Bhattacharyya AK, et al. Human sperm DNA integrity in normal and abnormal semen samples and its correlation with sperm characteristics. Andrologia 2009;41:207-15.

37. Chan PJ, Corselli JU, Patton WC, Jacobson JD, Chan SR, King A. A simple comet assay for archived sperm correlates DNA fragmentation to reduced hyperactivation and penetration of zona-free hamster oocytes. Fertil Steril 2001;75:186-92.

38. Boushaba S, Belaaloui G. Sperm DNA fragmentation and standard semen parameters in algerian infertile male partners. World J Mens Health 2015;33:1-7.

39. Virant-Klun I, Tomazevic T, Meden-Vrtovec H. Sperm singlestranded DNA, detected by acridine orange staining, reduces fertilization and quality of ICSI-derived embryos. J Assist Reprod Genet 2002;19:319-28.

40. Lewis $\mathrm{SE}$, Aitken RJ. DNA damage to spermatozoa has impacts on fertilization and pregnancy. Cell Tissue Res 2005;322:33-41.

41. Borini A, Tarozzi N, Bizzaro D, Bonu MA, Fava L, Flamigni C, et al. Sperm DNA fragmentation: paternal effect on early post-implantation embryo development in ART. Hum Reprod 2006;21: 2876-81. 
42. Lin MH, Kuo-Kuang Lee R, Li SH, Lu CH, Sun FJ, Hwu YM. Sperm chromatin structure assay parameters are not related to fertilization rates, embryo quality, and pregnancy rates in in vitro fertilization and intracytoplasmic sperm injection, but might be related to spontaneous abortion rates. Fertil Steril 2008;90:352-9.

43. Avendano C, Franchi A, Duran H, Oehninger S. DNA fragmentation of normal spermatozoa negatively impacts embryo quality and intracytoplasmic sperm injection outcome. Fertil Steril 2010; 94:549-57.

44. Enciso M, Pieczenik G, Cohen J, Wells D. Development of a novel synthetic oligopeptide for the detection of DNA damage in human spermatozoa. Hum Reprod 2012;27:2254-66.

45. Frydman N, Prisant N, Hesters L, Frydman R, Tachdjian G, CohenBacrie $\mathrm{P}$, et al. Adequate ovarian follicular status does not prevent the decrease in pregnancy rates associated with high sperm DNA fragmentation. Fertil Steril 2008;89:92-7.

46. Zini A, Boman JM, Belzile E, Ciampi A. Sperm DNA damage is associated with an increased risk of pregnancy loss after IVF and ICSI: systematic review and meta-analysis. Hum Reprod 2008; 23:2663-8.

47. Robinson L, Gallos ID, Conner SJ, Rajkhowa M, Miller D, Lewis S, et al. The effect of sperm DNA fragmentation on miscarriage rates: a systematic review and meta-analysis. Hum Reprod 2012; 27:2908-17.

48. Zidi-Jrah I, Hajlaoui A, Mougou-Zerelli S, Kammoun M, Meniaoui I, Sallem A, et al. Relationship between sperm aneuploidy, sperm DNA integrity, chromatin packaging, traditional semen parameters, and recurrent pregnancy loss. Fertil Steril 2016;105: 58-64.

49. Caglar GS, Koster F, Schopper B, Asimakopoulos B, Nehls B, Nikolettos $N$, et al. Semen DNA fragmentation index, evaluated with both TUNEL and Comet assay, and the ICSI outcome. In Vivo 2007;21:1075-80.

50. Nicopoullos JD, Gilling-Smith C, Almeida PA, Homa S, NormanTaylor JQ, Ramsay JW. Sperm DNA fragmentation in subfertile men: the effect on the outcome of intracytoplasmic sperm injection and correlation with sperm variables. BJU Int 2008;101: 1553-60.

51. Dar S, Grover SA, Moskovtsev SI, Swanson S, Baratz A, Librach CL. In vitro fertilization-intracytoplasmic sperm injection outcome in patients with a markedly high DNA fragmentation index (>50\%). Fertil Steril 2013;100:75-80.

52. Collins JA, Barnhart KT, Schlegel PN. Do sperm DNA integrity tests predict pregnancy with in vitro fertilization? Fertil Steril 2008;89:823-31.

53. Practice Committee of the American Society for Reproductive Medicine. The clinical utility of sperm DNA integrity testing. Fer- til Steril 2006;86(5 Suppl 1):S35-7.

54. Evenson D, Jost L. Sperm chromatin structure assay is useful for fertility assessment. Methods Cell Sci 2000;22:169-89.

55. Avendano C, Franchi A, Taylor S, Morshedi M, Bocca S, Oehninger $S$. Fragmentation of DNA in morphologically normal human spermatozoa. Fertil Steril 2009;91:1077-84.

56. Tamburrino L, Marchiani S, Montoya M, Elia Marino F, Natali I, Cambi $M$, et al. Mechanisms and clinical correlates of sperm DNA damage. Asian J Androl 2012;14:24-31.

57. Henkel R, Hajimohammad M, Stalf T, Hoogendijk C, Mehnert C, Menkveld $\mathrm{R}$, et al. Influence of deoxyribonucleic acid damage on fertilization and pregnancy. Fertil Steril 2004;81:965-72.

58. Hazout A, Dumont-Hassan M, Junca AM, Cohen Bacrie P, Tesarik J. High-magnification ICSI overcomes paternal effect resistant to conventional ICSI. Reprod Biomed Online 2006;12:19-25.

59. Lewis SE, John Aitken R, Conner SJ, Iuliis GD, Evenson DP, Henkel $\mathrm{R}$, et al. The impact of sperm DNA damage in assisted conception and beyond: recent advances in diagnosis and treatment. Reprod Biomed Online 2013;27:325-37.

60. Maettner R, Sterzik K, Isachenko V, Strehler E, Rahimi G, Alabart $J$, et al. Quality of human spermatozoa: relationship between high-magnification sperm morphology and DNA integrity. Andrologia 2014;46:547-55.

61. Osman A, Alsomait H, Seshadri S, El-Toukhy T, KhalafY. The effect of sperm DNA fragmentation on live birth rate after IVF or ICSI: a systematic review and meta-analysis. Reprod Biomed Online 2015;30:120-7.

62. Jiang $\mathrm{H}$, He RB, Wang $\mathrm{CL}$, Zhu J. The relationship of sperm DNA fragmentation index with the outcomes of in-vitro fertilisationembryo transfer and intracytoplasmic sperm injection. J Obstet Gynaecol 2011;31:636-9.

63. Larson-Cook KL, Brannian JD, Hansen KA, Kasperson KM, Aamold ET, Evenson DP. Relationship between the outcomes of assisted reproductive techniques and sperm DNA fragmentation as measured by the sperm chromatin structure assay. Fertil Steril 2003;80:895-902.

64. Benchaib M, Braun V, Lornage J, Hadj S, Salle B, Lejeune H, et al. Sperm DNA fragmentation decreases the pregnancy rate in an assisted reproductive technique. Hum Reprod 2003;18:1023-8.

65. Virro MR, Larson-Cook KL, Evenson DP. Sperm chromatin structure assay (SCSA) parameters are related to fertilization, blastocyst development, and ongoing pregnancy in in vitro fertilization and intracytoplasmic sperm injection cycles. Fertil Steril 2004;81:1289-95.

66. Seli E, Gardner DK, Schoolcraft WB, Moffatt O, Sakkas D. Extent of nuclear DNA damage in ejaculated spermatozoa impacts on blastocyst development after in vitro fertilization. Fertil Steril 
2004;82:378-83.

67. Wdowiak A, Bakalczuk S, Bakalczuk G. The effect of sperm DNA fragmentation on the dynamics of the embryonic development in intracytoplasmatic sperm injection. Reprod Biol 2015;15:94100.

68. Host $E$, Lindenberg S, Smidt-Jensen S. The role of DNA strand breaks in human spermatozoa used for IVF and ICSI. Acta Obstet Gynecol Scand 2000;79:559-63.

69. Tomlinson MJ, Moffatt O, Manicardi GC, Bizzaro D, Afnan M, Sakkas D. Interrelationships between seminal parameters and sperm nuclear DNA damage before and after density gradient centrifugation: implications for assisted conception. Hum Reprod 2001;16:2160-5.

70. Check JH, Graziano V, Cohen R, Krotec J, Check ML. Effect of an abnormal sperm chromatin structural assay (SCSA) on pregnancy outcome following (IVF) with ICSI in previous IVF failures. Arch Androl 2005;51:121-4.

71. Oleszczuk K, Giwercman A, Bungum M. Sperm chromatin structure assay in prediction of in vitro fertilization outcome. Andrology 2016;4:290-6.

72. Evenson D, Wixon R. Meta-analysis of sperm DNA fragmentation using the sperm chromatin structure assay. Reprod Biomed Online 2006;12:466-72.

73. Henkel R, Kierspel E, Hajimohammad M, Stalf T, Hoogendijk C, Mehnert C, et al. DNA fragmentation of spermatozoa and assisted reproduction technology. Reprod Biomed Online 2003;7:47784.

74. Van Dyk Q, Lanzendorf S, Kolm P, Hodgen GD, Mahony MC. Incidence of aneuploid spermatozoa from subfertile men: selected with motility versus hemizona-bound. Hum Reprod 2000;15: 1529-36.

75. Kumar D, Upadhya D, Uppangala S, Salian SR, Kalthur G, Adiga SK. Nuclear DNA fragmentation negatively affects zona binding competence of $Y$ bearing mouse spermatozoa. J Assist Reprod Genet 2013;30:1611-5.

76. Moskovtsev SI, Willis J, White J, Mullen JB. Sperm DNA damage: correlation to severity of semen abnormalities. Urology 2009; 74:789-93.

77. Khadem N, Poorhoseyni A, Jalali M, Akbary A, Heydari ST. Sperm DNA fragmentation in couples with unexplained recurrent spontaneous abortions. Andrologia 2014;46:126-30. 